\title{
Correction to: The Standard Model in noncommutative geometry: fundamental fermions as internal forms
}

\author{
Ludwik Dąbrowski ${ }^{1}$. Francesco D'Andrea ${ }^{2,3}$ (D) Andrzej Sitarz ${ }^{4,5}$
}

Published online: 28 August 2019

(c) Springer Nature B.V. 2019

\section{Correction to: Lett Math Phys https://doi.org/10.1007/s11005-017-1036-x}

The original version of the acknowledgement unfortunately contained a mistake. The grant number was not correct. The corrected grant number is given below.

2016/21/B/ST1/02438

Publisher's Note Springer Nature remains neutral with regard to jurisdictional claims in published maps and institutional affiliations.

The original article can be found online at https://doi.org/10.1007/s11005-017-1036-x.

\section{Francesco D'Andrea}

francesco.dandrea@unina.it

Ludwik Dąbrowski

dabrow@sissa.it

Andrzej Sitarz

sitarz@if.uj.edu.pl

1 Scuola Internazionale Superiore di Studi Avanzati (SISSA), via Bonomea 265, 34136 Trieste, Italy

2 Università di Napoli "Federico II", Via Cintia, 80126 Naples, Italy

3 I.N.F.N. Sezione di Napoli, Complesso MSA, Via Cintia, 80126 Naples, Italy

4 Instytut Fizyki Uniwersytetu Jagiellońskiego, Stanisława Łojasiewicza 11, 30-348 Kraków, Poland

5 IMPAN, ul. Śniadeckich 8, 00656 Warsaw, Poland 